\title{
Quercetin Dampens Postprandial Hyperglycemia in Type 2 Diabetic Patients Challenged with Carbohydrates Load
}

\author{
Hussain S. A. ${ }^{1, *}$, Ahmed Z. A. ${ }^{2}$, Mahwi T. O. ${ }^{3}$, Aziz T. A. ${ }^{4}$ \\ ${ }^{1}$ Department of Pharmacology and Toxicology, College of Pharmacy, University of Baghdad, Baghdad, Iraq \\ ${ }^{2}$ Department of Pharmacolo gy, School of Medicine, Faculty of Medical Sciences, University of Sulaimani, Kurdistan, Iraq \\ ${ }^{3}$ Department of Internal Medicine, School of Medicine, Faculty of Medical Sciences, University of Sulaimani, Kurdistan, Iraq \\ ${ }^{4}$ Department of Pharmacology, School of Pharmacy, Faculty of Medical Sciences, University of Sulaimani, Kurdistan, Iraq
}

\begin{abstract}
Postprandial hyperglycemia is a major risk factor for diabetic complications leading to disabilities and mortality in diabetics. Quercetin, a flavonoid, has been tried in traditional medicine for treating many disorders including diabetes. The present study was designed to evaluate the potential of quercetin to damp postprandial blood glucose level after maltose and glucose loading in patients with type 2 diabetes mellitus (DM). In a rando mized, b linded crossover study, single oral dose $(400 \mathrm{mg}$ ) of quercetin or placebo formula was ad min istered to the patients $30 \mathrm{~min}$ before loading with either maltose $(2 \mathrm{~g} / \mathrm{kg})$ or g lucose $(100 \mathrm{~g})$, blood samples are taken at different intervals for measurement of plas ma glucose. The results clearly showed ameliorated postprandial hyperglycemia due to the use of quercetin, it significantly dampened the postprandial hyperglycemia only after maltose loading compared to placebo; no effect reported for quercetin after glucose loading. In conclusion, quercetin effectively suppresses postprandial hyperglycemia in patients with type 2 DM loaded with maltose, which may be attributed to $\alpha$-glucosidase inhibition.
\end{abstract}

Keywords Quercetin, Type 2 DM, PPGE, Glucose Absorption

\section{Introduction}

Type 2 diabetes is characterized by two main features: peripheral insulin resistance and beta-cell dysfunction. Both hereditary and environmental factors, such as obesity and prolonged hyperglycemia, may trigger or exaggerate human type 2 diabetes. Hyperglycemia causes both beta-cell damage and peripheral insulin resistance via multiple mechanis ms, collectively referred to as glucotoxicity[1]. The acute glucose fluctuations during the postprandial period exhibits a more specific triggering effect on oxidative stress than chronic sustained hyperglycemia which suggests that the therapy in type 2 diabetes should target not only hemoglobin A 1c and mean glucose concentrations but also acute glucose swings[2]. In MODY-2 diabetes, functional defects in glucokinase genes restrict hepatic glucose uptake, bringing about prolongation of postprandial hyperglycemia[3], and eventually result in beta-cell overload.

Hence, efforts to minimize postprandial hyperglycemia, as well as fasting blood glucose control, are of importance for the prevention and treatment of type 2 diabetes. Oxidative stress, which is the result of either the overproduction of free

* Corresponding author:

saad_alzaidi@yahoo.com (Hussain HA)

Published online at http://journal.sapub.org/ diabetes

Copyright (C) 2012 Scientific \& Academic Publishing. All Rights Reserved radicals or some impairment in the cellular antioxidant systems, can lead to serious deterioration of health. For instance, oxidative stress is likely to be involved in both the development and the advancement of diabetes mellitus, as several studies have demonstrated the involvement of oxygen free radicals (reactive oxygen species) in the appearance of insulin resistance, a distinctive characteristic of type 2 diabetes[4,5]. In addition, oxidative stress might even play some direct role in the subsequent surfacing of other diabetes- related complications[6]. Quercetin $\left(3,3^{\prime}, 4^{\prime}, 5-7\right.$-penta-hydroxy flavone), is a flavonol chemically related to kaemferol. As a member of the flavonoid family, quercetin is widely distributed in plants, and is probably the most abundant of the flavonoid molecules in the plant kingdom. Sources of quercetin include brassica green vegetables, berries, onions, parsley, apple, legumes, green tea, citrus fruits, red grape wines, and so on[7]. Quercetin prevents oxidative injury and cell death[8] by several mechanisms, including scavenging oxygen radicals[9], inhibiting xanthine oxidase[10], lipid peroxidation, and chelating metal ions[11]. Quercetin is an efficient antioxidant, as evidenced by both in vitro[12] and in vivo studies[13], and in parallel, it has also been shown to improve diabetes-related impairments in animals $[14,15]$. Thus, in the present study, we address the question whether quercetin has beneficial effects in controlling and/or improving postprandial hyperglycemia in type $2 \mathrm{DM}$ patients challenged with mono- or disaccharide load. 


\section{Materials and Methods}

\subsection{Selection of Patients}

Twenty fourg libenclamide-treated type 2 diabetic patients were enrolled in this study. The study protocol was approved by the Ethical Committee of the College of Medicine/University of Sulaimani and carried out in accordance with the principles of the Declaration of Helsinki as revised in 2000, and all patients gave informed consent. No subjects were taking any medications that influence glycemic control other than glibenclamide, and all abstained fro m a lcohol, tobacco, and strenuous physical activity for 24 hrs and caffeine-containing drinks overnight.

\subsection{Study Protocol}

The patients were randomized into two groups of (12 in each), the effect of either placebo formula or single $400 \mathrm{mg}$ quercetin dihydrate oral dose on postprandial glucose excursions after loading with either $2 \mathrm{~g} / \mathrm{kg}$ maltose or $100 \mathrm{~g}$ glucose were evaluated in a crossover design. After overnight fasting, either single $400 \mathrm{mg}$ quercetin dihydrate (Xian Keen, China), specially formulated for this purpose, or placebo was orally administered, followed (after $30 \mathrm{~min}$ ) by loading of maltose or glucose. The order to receive capsules (placebo or quercetin) was randomized, and the kind of capsules taken was blinded to the examiner. Blood samples were obtained at $0,30,60,90,120,150$ and $180 \mathrm{~min}$ in tubes containing EDTA sodium $(1 \mathrm{mg} / \mathrm{ml})$ and immediately separated for plas ma by centrifugation at $3000 \mathrm{rpm}$ at $4^{\circ} \mathrm{C}$ for $10 \mathrm{~min}$. Routine chemical methods were used to determine the plasma concentrations of glucose[16].

\subsection{Statistical Analysis}

Values are expressed as the mean $\pm \mathrm{SD}$. Area under the curve (AUC) that represents the relation between the change in plasma glucose with respect to baseline and time was estimated. Two-tailed unpaired Student's $t$ test or one-way factorial ANOVA, followed by Bonferroni's post hoc comparisons, was used to compare means of AUC. $P<0.05$ was considered statistically significant. Analyses were processed using GraphPad Prism software for W indows (version 5.0, GraphPad Software, Inc., San Diego, CA).

\section{Results}

Figure 1 showed that quercetin did not produce apparent changes on the rate of postprandial hyperglycemia after glucose load, where the changes in plasma glucose levels appeared comparable in both quercetin and placebo treated groups. Meanwhile, figure 2 clearly showed that adminis tration of $400 \mathrm{mg}$ quercetin, as single oral dose before maltose challenge, decreased the magnitude of glucose spikes during 3 hrs follow up of glucose levels compared to placebo group.

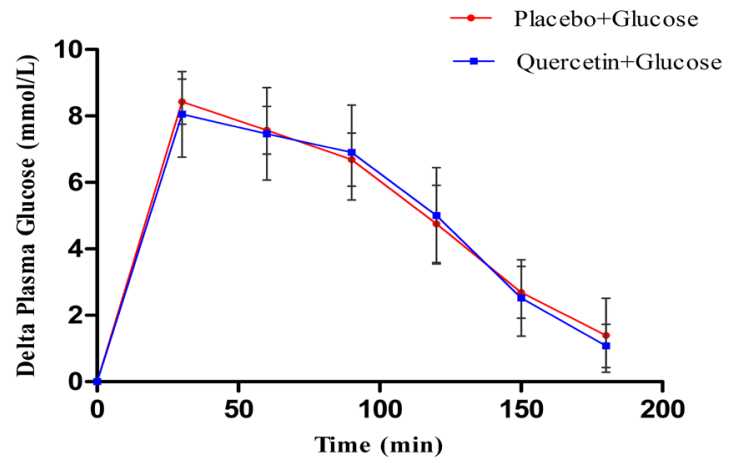

Figure 1. Effect of single oral dose $(400 \mathrm{mg})$ of quercet in and placebo on the change in plasma glucose levels after $100 \mathrm{~g}$ glucose load in type $2 \mathrm{DM}$ patients

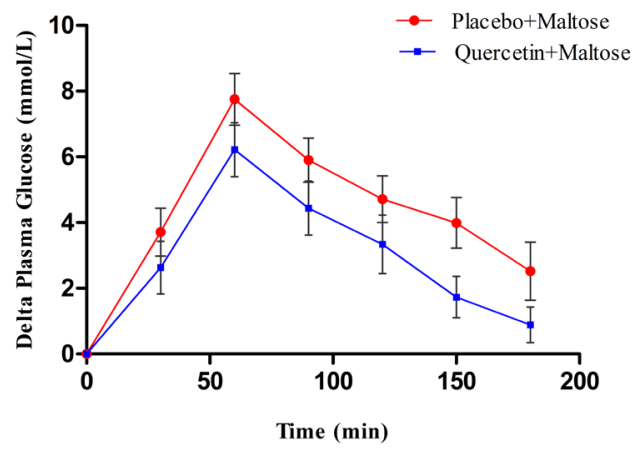

Figure 2. Effect of single oral dose $(400 \mathrm{mg})$ of quercet in and placebo on the change in plasma glucose levels after $2 \mathrm{~g} / \mathrm{kg}$ maltose load in type $2 \mathrm{DM}$ patients

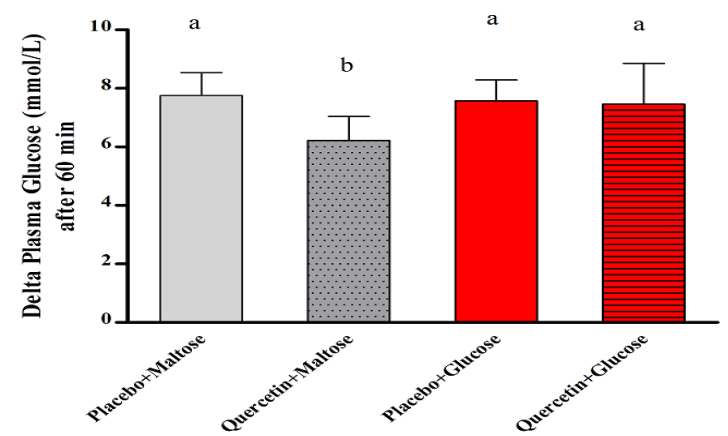

Figure 3. Effect of single oral dose $(400 \mathrm{mg})$ of quercet in and placebo on the plasma glucose excursions (at $60 \mathrm{~min}$ interval) after either $2 \mathrm{~g} / \mathrm{kg}$ maltose or $100 \mathrm{~g}$ glucose load in type $2 \mathrm{DM}$ patients; values with non ident ical letters $(\mathrm{a}, \mathrm{d})$ are considered significantly different $(P \varangle 0.05)$

When the mean changes in plasma glucose level after 60 min of carbohydrate challenge were compared, quercetin produced significant reduction $(P<0.05)$ only after maltose challenge compared to other groups (Figure 3 ). In figure 4, the area under the curve (AUC) for postprandial hyperglycemia, during $3 \mathrm{hrs}$ after challenge with either glucose or maltose load, clearly indicated that quercetin significantly dampen postprandial hyperglycemia only after maltose challenge, while no such effect reported after administration of glucose. 


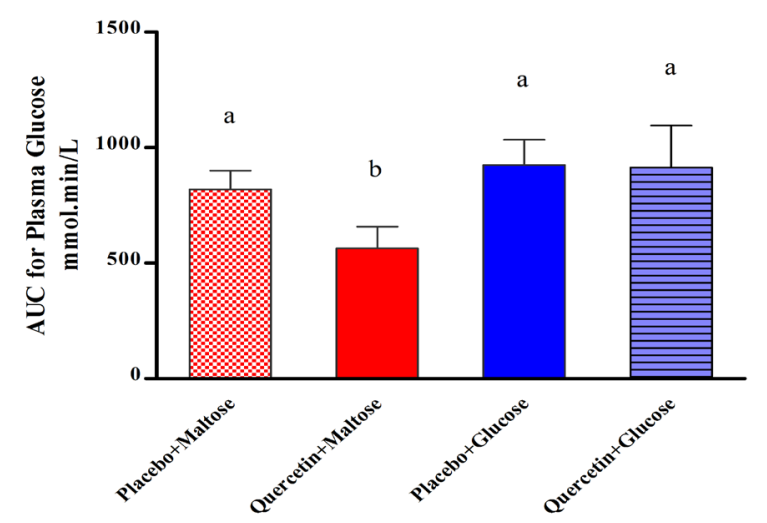

Figure 4. Effect of single oral dose $(400 \mathrm{mg})$ of quercet in and placebo on the $\mathrm{AUC}_{1-180 \mathrm{~min}}$ for the change in plasma glucose levels after either $2 \mathrm{~g} / \mathrm{kg}$ maltose or $100 \mathrm{~g}$ glucose load in type $2 \mathrm{DM}$ patients; values with non identical letters $(a, d)$ are considered significantly different $(P \triangleleft 0.05)$

\section{Discussion}

Controlling not only fasting, but also postprandial hyperglycemia, is important in achieving tight control of blood glucose levels, which is the major target of diabetic therapy[17]. It was reported that postprandial hyperglycemia might be more strongly correlated with cardiovascular morbidity and mortality than fasting hyperglycemia[18]. Postprandial hyperglycemia has been shown to increase the production of free radicals, which induce vasoconstriction and stimulate prothrombotic pathways leading to an increased risk of card iovascular disease, the major cause of premature death among type 2 diabetic patients[19]. Although $\alpha$-glucosidase inhibitors are common oral hypoglycemic agents, the chronic use of these agents can lead to gastrointestinal side effects such as flatulence, vomiting, and diarrhea[20]. Thus, many investigators have performed studies to discover $\alpha$-glucosidase inhibitors from natural products with reduced side effects[21,22]. A mong known materials with $\alpha$-glucosidase inhibitor activity, guava leaf extract and Touchi extract have been approved as individual authorized health functional foods to improve postprandial hyperglycemia in Korea, and as Foods for Specified Health Use (FOSHU) in Japan[23]. However, few flavonoid drugs have reached clinical application so far, mostly due to the ple iotropic effects inherent to this class of substances and difficulties to anticipate side-effects. Quercetin is an exception since it has already been used therapeutically for a long time. Thus, there is considerable amount of data about its efficiency, pharmacokinetics, and safety[24]. In the present study, the data indicated that quercetin can decrease postprandial glucose level after disaccharides loading, which may be mainly attributed to inhibition of $\alpha$-glucosidase as one of the expected mechanis ms for the reduction of plasma glucose; this effect subsequently lead to suppression of postprandial hyperglycemia. Thus, quercetin can be considered as a potential candidate for the management of type 2 diabetes mellitus by this mechanism. Medicines that reduce postprandial hyperglycemia by suppressing the absorption of carbohydrates are shown to be effective for prevention and treatment of non-insulin-dependent diabetes mellitus[25]. Many naturally occurring flavonoids have hypoglycemic potential and have been described as glucosidase inhibitors; they are considered as a promising source for drug development and have shown impressing biological effects in vitro and in vivo[26]. While the antioxidant activity is regarded as the main mechanis $m$ underlying the biological effects of quercetin in many diseases, recent evidence suggests that quercetin could be benefic ial in the treatment of type 2 diabetes owing to its anti-hyperglycemic properties[15]. Recent studies have shown that many flavonoids including quercetin and cyanidin-3-g lucoside inhibit in vitro the intestinal $\alpha$-glucosidase activity[27,28]. Considering the data obtained from our investigation, quercetin may play similar role in controlling postprandial hyperglycemia by inhibiting intestinal $\alpha$-glucosidase in humans with type $2 \mathrm{DM}$. According to literature evaluation about flavonols and flavones, it can be assumed that quercetin may interact with protein by using hydroxyl groups in their molecular structure, resulting in the formation of hydrogen bonds with the polar groups (amide, guanidine, peptide, amino and carboxyl groups) in the active site of proteins by covalent and/or non-covalent interactions. To prove this hypothesis, X-ray crystallography and computer modeling to evaluate the binding activity of quercetin on intestinal $\alpha$-glucosidase is needed for further investigation. Although further study is required for the kinetic of the enzyme inhibition, encouraging data were previously available concerning the inhibitory effects of many flavonoids on $\alpha$-glucosidase enzyme; although these findings were incomplete, but they can describe the hypoglycemic effect of flavonoid by inhibition of intestinal glucosidase activity[26]; accordingly, the finding of the present study is compatible with this assumption. We have also evaluated the effect of quercetin on glucose loading in diabetic patients. Quercetin, when admin istered as single oral dose, do not suppress the postprandial hyperglycemia associated with glucose loading similar to that of maltose loading; this indicates that the major mechanis m of action of postprandial glucose suppression is attributed only to the inhibition of $\alpha$-glucosidase, but the involvement of other mechanis ms can not be ruled out when multiple doses used for longer period of time. The reported results of the current study may provide preliminary evidence about quercetin action that could serve as a base for a pilot clinical study in this respect. In the present study, the observed reduction in maltose-induced postprandial hyperglycemia following treatment of type 2 diabetic patients with quercetin, confirm the potential role of this flavonoid to inhibit intestinal $\alpha$-glucosidase activity; the failure of quercetin to damp glucose-induced glycemic spikes confirm the involvement of this mechanism in controlling postprandial hyperglycemia. In conclusion: orally ad min istered quercetin as a single dose in patients with type 2 DM dampen postprandial 
hyperglycemia induced by maltose load, while has no effect when diabetic patients are loaded with glucose.

\section{ACKNOWLEDGEMENTS}

The presented data were abstracted from $\mathrm{PhD}$ thesis submitted to the Department of Pharmacology, School of Medicine, University of Sulaimani. The authors thank University of Sulaimani for supporting the project

\section{REFERENCES}

[1] Bonora, E. (2008). Protection of pancreatic beta-cells: is it feasible? Nutr. Metab. Cardiovasc. Dis., 18:74-83.

[2] Monnier, L. and Colette, C. (2006). Contributions of fasting and postprandial glucose to hemoglobin A1c. Endocr. Pract., 12(Supp1 1):42-46.

[3] Jiang, M.H.; Fei, J.; Lan, M.S.; et al. (2008). Hypermethylation of hepatic Gck promoter in ageing rats contributes to diabetogenic potential. Diabetologia, 51:1525-1533.

[4] Delbosc, S.; Paizanis, E.; Magous, R.; et al. (2005). Involvement of oxidative stress and NADPH oxidase activation in the development of cardiovascular complications in a model of insulin resistance, the fructose-fed rat. Atherosclerosis, 179:43-49.

[5] Wei, Y.; Whaley-Connell, A.T.; Chen, K.; et al. (2007). NADPH oxidase contributes to vascular inflammation, insulin resistance, and remodeling in the transgenic (mRen2) rat. Hypertension, 50:384-391.

[6] Evans, J.L.; Maddux, B.A. and Goldfine, I.D. (2005). The molecular basis for oxidative stress-induced insulin resistance. Antioxid. Redox. Signal., 7:1040-1052.

[7] Hakkinen, S.H.; Karenlampi, S.O.; Heinonen, I.M.; et al. (1999). Content of the flavonols quercetin, myricetin, and kaempferol in 25 edible berries. J. Agric. Food Chem., 47:2274-2279.

[8] Larocca, L.M.; Teofili, L.; Sica, S.; et al. (1995). Quercetin inhibits the growth of leukemic progenitors and induces the expression of transforming growth factor-B1 in these cells. Blood, 85:3654-3661.

[9] Cox, D.; Whichelow, M.J. and Prevost, T.A. (2000). Antioxidant effects of flavonoids. Public Health Nutr., 3:19-29.

[10] Chang, W.S.; Lee, Y.J.; Lu, F.J. and Chiang, H.C. Inhibitory effects of flavonoids on xanthine oxidase. Anticancer Res., 13:2165-2170.

[11] Chen, Y.T.; Zheng, R.L.; Jia, Z.J. and Ju, Y. (1990). Flavonoids as superoxide scavengers and antioxidants. Free Radic. Biol. Med., 9:19-20.

[12] Fiorani, M.; Guidarelli, A.; Blasa, M.; et al. (2010) Mitochondria accumulate large amounts of quercetin: prevention of mitochondrial damage and release upon oxidation of the extra-mitochondrial fraction of the flavonoid. J. Nutr. Biochem. 21:397-404.
[13] Meyers, K.J.; Rudolf, J.L. and Mitchell, A.E. (2008). Influence of dietary quercetin on glutathione redox status in mice. J. A gric. Food Chem., 56:830-836.

[14] Kobori, M.; Masumoto, S.; Akimoto, Y. and Takahashi, Y. (2009). Dietary quercetin alleviates diabetic symptoms and reduces streptozotocin-induced disturbance of hepatic gene expression in mice. Mol. Nutr. Food Res., 53:859-868.

[15] Youl, E.; Bardy, G.; Magous, R.; et al. (2010). Quercetin potentiates insulin secretion and protects INS-1 pancreatic $\beta$-cells against oxidative damage via the ERK1/2 pathway. Br. J. Pharmacol., 161:799-814.

[16] Barham, D. and Trinder, P. (1972). An improved color reagent from the determination of blood glucose by the oxidative system. Analyst, 97:142-145.

[17] Abrahamson, M.J. (2004). Optimal gly cemic control in type 2 diabetes mellitus: fasting and postprandial glucose in context. Arch. Intern. Med., 164:486-491.

[18] Haller, H. (1998). The clinical importance of postprandial glucose. Diabetes Res. Clin. Pract., 40:S43-S49.

[19] Ceriello, A.; Davidson, J.; Hanefeld, M.; et al. (2006). Postprandial hyperglycemia and cardiovascular complications of diabetes: an update. Nutr. Metab. Cardiovasc. Dis., 16:453-456.

[20] Hanefeld, M. (1998). The role of acarbose in the treatment of non-insulin-dependent diabetes mellitus. J. Diab. Complications, 12:228-237.

[21] Gholamhoseinian, A.; Fallah, H. and Sharififar, F. (2009). Inhibitory effect of methanol extract of Rosa damascena Mill. flowers on alpha-glucosidase activity and postprandial hyperglycemia in normal and diabetic rats. Phytomedicine, 16:935-41.

[22] Wang, H.; Du, Y.J. and Song, H.C. (2010). $\alpha$-Glucosidase and $\alpha$-amylase inhibitory activities of guava leaves. Food Chem., 123:6-13.

[23] Saito, M. (2007). Role of FOSHU (food for specified health uses) for healthier life. Yakugaku Zasshi, 127:407-416.

[24] Erlund, I.; Freese, R.; Marniemi, J.; et al. (2006). Bioavailability of quercetin from berries and the diet. Nutr. Cancer, 54:13-17.

[25] Matsuda, H.; Morikawa, T. and Yoshikawa, M. (2002). Antidiabetogenic constituents from several natural medicines. Pure Appl. Chem., 74(7):1301-1308.

[26] Pereira, D.F.; Cazarolli, L.H.; Lavado, C.; et al. (2011). Effects of flavonoids on $\alpha$-glucosidase activity: potential targets for glucose homeostasis. Nutrition, 27(11-12):1161-1167.

[27] Jo, S.H.; Ka, E.H.; Lee, H.S.; et al. (2009). Comparison of antioxidant potential and rat intestinal $\alpha$-glucosidases inihibitory activities of quercetin, rutin, and isoquercetin. Int. J. Appl. Res. Nat. Prod., 2:52-60.

[28] Akkarachiyasit, S.; Charoenlertkul, P.; Yibchok-anun, S. and Adisakwattana, S. (2010). Inhibitory activities of cyanidin and Its gly cosides and synergistic effect with acarbose against intestinal $\alpha$-glu cosidase and pancreatic $\alpha$-amy lase. Int. J.Mol. Sci., $11: 3387-3396$. 